\title{
Exciton Polaritons in a Two-Dimensional Lieb Lattice with Spin-Orbit Coupling
}

\author{
C. E. Whittaker, ${ }^{1, *}$ E. Cancellieri, ${ }^{1,3}$ P. M. Walker, ${ }^{1}$ D. R. Gulevich,${ }^{2}$ H. Schomerus ${ }^{3}$ D. Vaitiekus, ${ }^{1}$ \\ B. Royall, ${ }^{1}$ D. M. Whittaker, ${ }^{1}$ E. Clarke, ${ }^{4}$ I. V. Iorsh, ${ }^{2}$ I. A. Shelykh, ${ }^{2,5}$ \\ M. S. Skolnick, ${ }^{1,2}$ and D. N. Krizhanovskii ${ }^{1,2,}$ \\ ${ }^{1}$ Department of Physics and Astronomy, University of Sheffield, Sheffield S3 7RH, United Kingdom \\ ${ }^{2}$ ITMO University, St. Petersburg 197101, Russia \\ ${ }^{3}$ Department of Physics, Lancaster University, Lancaster LA1 4YB, United Kingdom \\ ${ }^{4}$ EPSRC National Centre for III-V Technologies, University of Sheffield, Sheffield S1 3JD, United Kingdom \\ ${ }^{5}$ Science Institute, University of Iceland, Dunhagi 3, IS-107, Reykjavik, Iceland
}

(Received 4 May 2017; revised manuscript received 9 October 2017; published 2 March 2018)

\begin{abstract}
We study exciton polaritons in a two-dimensional Lieb lattice of micropillars. The energy spectrum of the system features two flat bands formed from $S$ and $P_{x, y}$ photonic orbitals, into which we trigger bosonic condensation under high power excitation. The symmetry of the orbital wave functions combined with photonic spin-orbit coupling gives rise to emission patterns with pseudospin texture in the flat band condensates. Our Letter shows the potential of polariton lattices for emulating flat band Hamiltonians with spin-orbit coupling, orbital degrees of freedom, and interactions.
\end{abstract}

DOI: 10.1103/PhysRevLett.120.097401

Two-dimensional lattices with flat energy bands attract keen research interest as a platform for studying exotic many-body effects including itinerant ferromagnetism [1], Wigner crystallization [2], and fractional quantum Hall phases [3]. A notable example of a flat-band system is the Lieb lattice [4], a decorated square lattice found in nature in the cuprates exhibiting high- $T_{c}$ superconductivity [5] and studied extensively in recent years for its topologically nontrivial phases [6-16]. In bosonic systems, models of particles in two-dimensional Lieb lattice potentials with flat energy bands are a highly valuable tool for researchers, having recently been experimentally realized in photonic waveguide arrays [17-20] and ultracold atoms in optical lattices [21]. Particularly fascinating prospects which remain unexplored in Lieb lattice models are many-body interactions, spin-orbit coupling (SOC) terms, and orbital structure. With such features, the flat bands are predicted to support nonlinear compactons [22,23] and interactioninduced topological phases [16]. More generally, these lattices allow one to study the interplay between fundamental nonlinear, spin, and orbital phenomena in a topological system.

Exciton-polariton (polariton) gases confined in lattice potentials have recently emerged as an attractive candidate for emulating nonlinear lattice Hamiltonians [24]. Microcavity polaritons are the mixed light-matter eigenmodes characterized by a small effective mass, allowing both quasiequilibrium and nonequilibrium Bose-Einstein condensation at elevated temperatures [25-28]. Giant exciton-mediated Kerr nonlinearity, which is 3-4 orders of magnitude larger than light-matter systems in the weakcoupling regime [29], has enabled the observation of ultralow power solitons [30] and vortices [31], and more recently, driven-dissipative phase transitions associated with quantum fluctuations [32-34]. In polariton systems, straightforward optical techniques can be used to create interacting scalar and spinor boson gases in highly tunable lattice geometries, which can be engineered through modulation of the photonic [35-37] or excitonic [38-41] potential landscape. Furthermore, the spatial, spectral, and pseudospin (polarization) properties of the polaritonic wave functions are directly accessible due to the finite cavity photon lifetime. The inherently nonequilibrium nature of polariton gases also means that higher energy orbital bands, formed from spatially anisotropic modes, are readily populated, as was recently demonstrated in a honeycomb lattice [42].

One intriguing property of polaritons in lattices is polarization-dependent tunneling [43], inherited mostly from the photonic component and enhanced by TE-TM splitting. It is formally analogous to SOC [43-45] inducing a $k$-dependent effective magnetic field acting on polariton pseudospin. The rich variety of polarization phenomena exhibited by polaritons in both noninteracting and interacting regimes [46] remains unexplored in two-dimensional periodic potentials, and is inaccessible in the inherently asymmetric one-dimensional case [47].

In this Letter, we study a two-dimensional (2D) array of coupled micropillars arranged in a Lieb lattice. The crystal structure comprises three square sublattices (denoted $A, B$, and $C$ ) each contributing one atom to the unit cell [Fig. 1(a)]. This lattice topology, in which the sites on different sublattices have different connectivity, results in localized states residing on dispersionless energy bands [48]. Here, we 
explore the bands formed by evanescent coupling of both the ground and first excited states of the pillars, which are 2D photonic orbitals with $S$ - and $P$-like wave functions. We excite the system quasiresonantly to optically load polaritons into the periodic potential, triggering condensation into three separate modes of the lattice $-S$ and $P$ flat (nonbonding) bands and the maxima of the $S$ antibonding (AB) band. Resolving the near-field emission in energy and polarization above the threshold for polariton condensation, we see that the flat band condensates show novel pseudospin textures arising from a polarization-dependent hopping energy, which acts as SOC for polaritons. Significant variation in the emission energy across real space (fragmentation) can be seen in the flat-band condensates, which we show arises due to the effect of many-body interactions, since the kinetic energy scale is quenched. This contrasts with the condensates formed on the $S \mathrm{AB}$ (dispersive) band which emit at strictly one energy.

Our 2D Lieb lattice consists of AlGaAs/GaAs micropillars of $3 \mu \mathrm{m}$ diameter and a separation of $2.9 \mu \mathrm{m}$. The lattice periodicity is $\alpha=5.8 \mu \mathrm{m}$. Further details about the sample and experimental methods can be found in the Supplemental Material [49]. The single-particle band structure of our Lieb lattice at $k_{x}=\pi / \alpha$ is displayed in Fig. 1(b). It shows the energy bands associated with the two lowest energy pillar modes, $S$ and $P$ (comprising degenerate $P_{x}$ and $P_{y}$ ) orbitals which have bare energies of around 1.4642 and $1.4662 \mathrm{eV}$, respectively. Photonic coupling results in $S$ - and $P$-type flat bands [(c) and (e) in Fig. 1(b)] separated by $2 \mathrm{meV}$ and a forbidden energy gap of approximately $0.8 \mathrm{meV}$ between $S$ and $P$ dispersive bands. The white dotted lines in Fig. 1(b) correspond to the

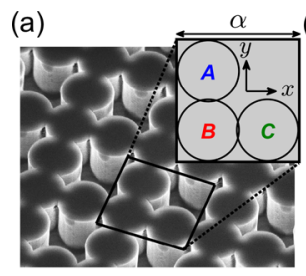

(c)
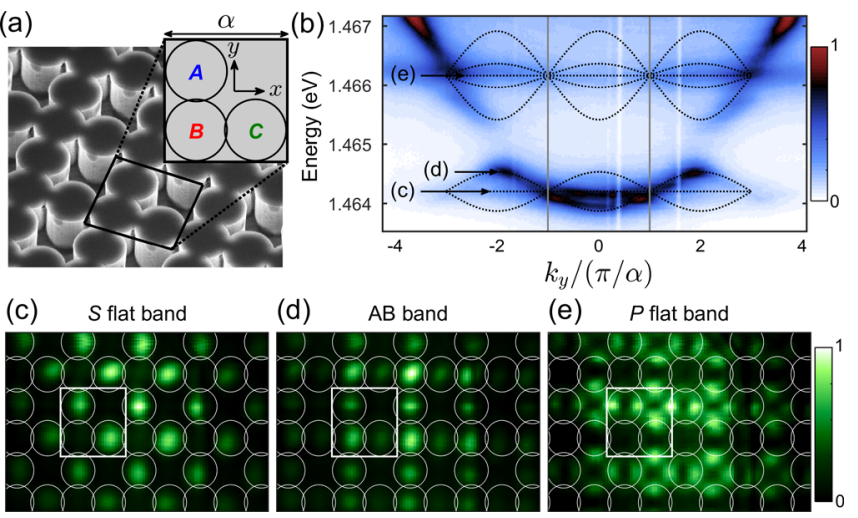

(e)

e) $\quad P$ flat band

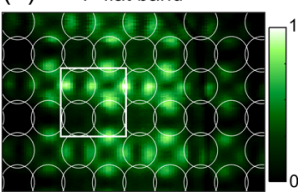

FIG. 1. (a) Scanning electron microscope image of a section of the 2D Lieb lattice. The enlarged image shows a schematic diagram of 1 unit cell and the 3 sublattices. (b) Lattice emission in energy-momentum space measured under low-power nonresonant excitation. White dotted lines correspond to bands calculated from the tight-binding model. (c)-(e) Emission in real space at the energies of the $S$ flat band (c), the AB band maxima (d) and the $P$ flat band (e). The white squares correspond to one unit cell as shown in (a) and the corresponding energies are shown by arrows in (b). dispersion curves calculated from our tight-binding (TB) model [49]. Experimentally, the emission from some of the expected folded branches of the dispersion appears almost absent. For example, emission from the $S \mathrm{AB}$ band [(d) in Fig. 1(b)] is suppressed within the first Brillouin zone (delimited by vertical lines) while the $S$ bonding band is suppressed outside. This effect can be attributed to a combination of far-field destructive interference and varying lifetimes (due to relaxation and losses) of different modes. This effect is well known in honeycomb lattices [36,51], and in Ref. [49], we solve the 2D Schrödinger equation for a periodic potential to confirm that this is also the case for the Lieb lattice.

In Figs. 1(c) and 1(e), we show the real space distribution of polariton emission intensity of the $S$ and $P$ flat bands, constructed by scanning the emission across the spectrometer slit and piecing together the energy-resolved slices. In both cases, there is highly suppressed emission from the $B$ sublattice, characteristic of flat bands, indicating that polaritons are highly localized on $A$ and $C$ sublattices. This results from destructive wave interference of the $A$ and $C$ sublattice linear eigenmodes due to the local lattice symmetries [52]. Interestingly, for the $P$ band, we see that emission from $P_{x}$ orbitals dominates on the $A$ sites and $P_{y}$ orbitals for the $C$ sites (the subscript denotes the axis to which the two lobes lie parallel). Since orthogonal $P$ orbitals do not interfere with each other, the absence of emission from $B$ sites must arise from destructive interference of like $P$ orbitals. A qualitative explanation is that the difference in the tunneling energies for spatially anisotropic modes with orthogonal orientations ( $\sigma$ and $\pi$ bonding) is offset by a difference in the orbital populations on the two sites maintaining the destructive interference necessary for flat band formation. We expand this argument including polarization later in the text. In contrast to the two flat bands, at the energy maxima of the dispersive $A B$ mode [Fig. 1(d)] the polaritons are delocalized across all three sublattices as is usually expected for the linear eigenmodes of a periodic potential.

In order to study our system in the nonlinear kinetic condensation regime, we tune the pump laser to $843 \mathrm{~nm}$, resonant with high energy states of the lower polaritonic bands (detuned roughly $-1 \mathrm{meV}$ from the exciton) where a broad continuum of high energy pillar modes exists [49]. Through this channel, we resonantly inject polaritons into the lattice at normal incidence, using high irradiances to create large populations of interacting polaritons. We use a large pump spot $(\sim 25 \mu \mathrm{m})$ which excites around 15 unit cells of the lattice. In Fig. 2, we show the evolution of our system with sample irradiance. Figures 2(c)-2(g) show the momentum space emission at $k_{x}=0$. Beyond a critical pumping intensity, macroscopic populations of particles begin to accumulate in the $P$ flat band as evidenced by a superlinear increase in the emission intensity [Fig. 2(a)] and narrowing of the linewidth [Fig. 2(b)] which 


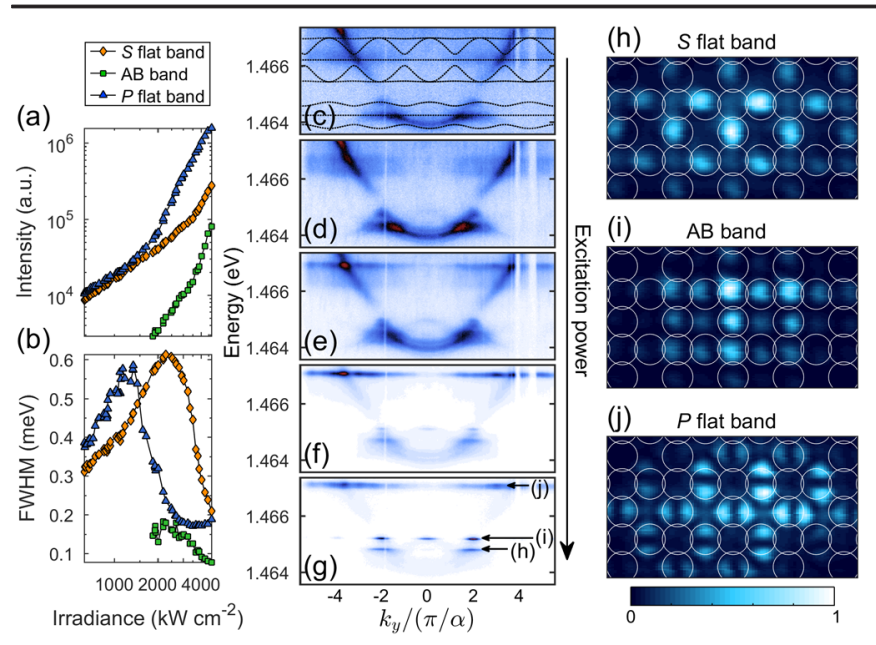

FIG. 2. (a) Peak intensity and (b) full width at half maximum of the three lattice modes as a function of the sample irradiance in the vicinity of the condensation thresholds. (c)-(g) The far-field emission with increasing irradiance, with condensation occurring in (f) and (g). The sample irradiances are 620 (c), 1360 (d), 2180 (e), 4030 (f), and $5630 \mathrm{~kW} \mathrm{~cm}^{-2}$ (g). The color scale is the same as that of Fig. 1(b). (h)-(j) Real space images of the lattice condensates. a.u. refers to arbitrary units.

signifies increased temporal coherence [27,53]. A similar condensation process is seen at slightly higher pumping intensities for the $S \mathrm{AB}$ band maxima, which move into the spectral gap, and the $S$ flat band. As can be seen in Figs. 2(f) and 2(g), these coexisting condensates dominate the normalized PL spectra above threshold. In the $S \mathrm{AB}$ band, condensates are formed in the negative effective mass states with an energy residing in the forbidden gap, which is reminiscent of the gap solitons previously reported as nonlinear solutions in similarly shallow periodic potentials $[54,55]$. The real space distributions of the three condensate modes are shown in Figs. 2(h)-2(j). The dark $B$ sites observed for the flat band cases confirm that the condensates, indeed, reside on highly nondispersive energy bands, in contrast to the condensates formed at the maxima of the dispersive $A B$ band.

Above the condensation threshold, the high density of polaritons leads to a sizable mean-field interaction energy due to Coulomb interactions between polaritons residing in the condensates as well as interactions of condensed polaritons with the highly populated resonantly pumped states. For the case of flat bands, the kinetic energy scale is quenched due to an infinite effective mass, so energy renormalization is nontrivial and cannot be treated as a perturbation [56]. With no kinetic energy to counterbalance the local nonlinear interaction energy, a fragmentation of the condensates into localized modes emitting at slightly different energies is observed. Conversely, the $\mathrm{AB}$ band polaritons acquire kinetic energy when they propagate from high to low density regions compensating the low potential energy in low density regions, resulting in a homogeneous

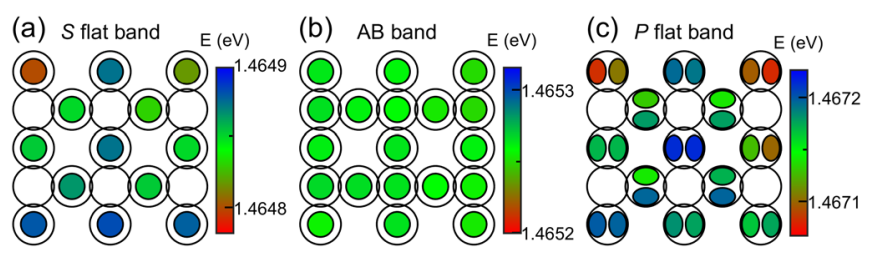

FIG. 3. (a) Color maps showing spatial energy variation of the $S$ flat band (a), AB band (b), and $P$ flat band (c) condensate emission constructed from experimental data above threshold.

emission energy across the lattice in real space. Spatial maps constructed from experimental data above threshold are shown in Fig. 3 and demonstrate the degree of spectral variation of the three condensed modes, which is vanishing for the $\mathrm{AB}$ band but pronounced for the flat bands. In Ref. [49], we analyze the relation between the population and energy across the condensates and show correlations which provide further evidence for the strong influence of many-body interactions in the fragmentation of the flat energy bands.

So far, we have studied the spatial and spectral properties of polaritons in both dispersive and flat bands, demonstrating bosonic condensation and analyzing the effect of nonperturbative many-body interactions. Now, we consider the pseudospin degree of freedom by resolving the lattice emission in polarization. In Figs. 4(a) and 4(b), we plot the Stokes linear polarization parameter $S_{1}$ for the flat band condensates, with $S_{1}=\left(I_{H}-I_{V}\right) /\left(I_{H}+I_{V}\right)$ where $I_{H}$ and $I_{V}$ are the intensities of the emitted light measured in the horizontal $\left(0^{\circ}\right)$ and vertical $\left(90^{\circ}\right)$ bases, respectively. Ordered pseudospin textures extended across several unit cells can be seen. The finite spatial extent of the pump spot and the intensity-dependent blueshift associated with its Gaussian profile limits the size of the observed patterns.

(a)
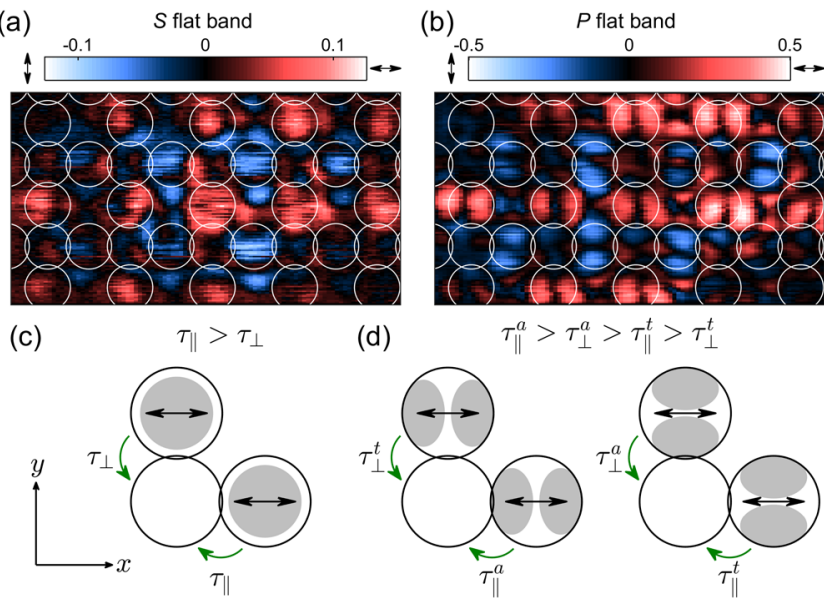

(d) $\tau_{\|}^{a}>\tau_{\perp}^{a}>\tau_{\|}^{t}>\tau_{\perp}^{t}$
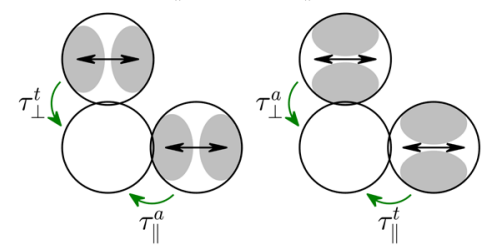

FIG. 4. $S_{1}$ linear Stokes parameter of the real space emission at the energies of the $S$ (a) and $P$ (b) flat band. The color scale is linear with red (blue) representing $H(V)$ polarization, depicted by arrows on either side of the color bar. (c),(d) Schematic of the nearest neighbor hopping processes for $S, P_{x}$, and $P_{y}$ orbitals, in the case of polarization-dependent hopping probabilities. 
In order to explain these polarization patterns, one needs to consider the two following features of Lieb lattices and Bragg-cavity polariton systems. First, in Lieb lattices the eigenmodes associated with the flat bands are nonspreading modes characterized by having zero population on the $B$ sites. This characteristic feature is due to the destructive interference of particles tunneling to $B$ sites from the neighboring $A$ and $C$ sites [52]. Clearly, in the case of different tunneling probabilities, the destructive interference can occur only if the neighboring sites have different populations, since the number of particles tunneling from one site to another is proportional both to the tunneling probability and to the number of particles on an initial site.

Second, in lattices formed from Bragg cavities, the particles' tunneling probability from one pillar to another has been experimentally observed [43] and theoretically discussed [57] to be polarization-dependent, leading to effective spin-orbit coupling. In particular, it has been shown that the tunneling probability $\left(\tau_{\|}\right)$of particles having polarization parallel to the propagation direction is higher than the tunneling probability $\left(\tau_{\perp}\right)$ of particles having polarization perpendicular to the propagation direction

$$
\tau_{\|}>\tau_{\perp}
$$

In the case of the Lieb lattice, this means that horizontally $(H)$ polarized particles tunnel with probability $\tau_{\|}$ between $B$ and $C$ sites and with probability $\tau_{\perp}$ between $B$ and $A$ sites, since the projection of the polarization is longitudinal and transverse to the tunneling directions, respectively [see Fig. 4(c)]. This means that the $H$-polarized eigenmodes of the $S$ flat band must have a higher population on $A$ sites, to compensate for the lower tunneling probability [Eq. (1)]. The opposite holds for vertically $(V)$ polarized eigenmodes, which will be characterized by a higher population on $C$ sites.

Finally, in our case, the quasiresonant pump populates both linear combinations of $\mathrm{H}$-polarized eigenmodes and linear combinations of $V$-polarized eigenmodes of the $S$ band. This, combined with the different populations of differently polarized particles results in a nonzero degree of polarization on $A$ and $C$ sites. In order to confirm this explanation, we developed a TB model with polarizationdependent tunneling terms [49]. By fitting the energy width of the $S$ band and the degree of polarization of the two sublattices, we deduce the two hopping parameters to be $\tau_{\|}=0.165 \mathrm{meV}$ and $\tau_{\perp}=0.145 \mathrm{meV}$, in agreement with previous experimental results [43]. With these values, we obtain a degree of polarization of the order of 0.128 , in excellent agreement with the experimental value of 0.13 .

For the $P$ flat band, the polarization pattern is qualitatively the same as that of the $S$ flat band albeit with a higher degree of polarization. It can be explained in the same way. In this case, however, one also has to consider that the $P$ state of each pillar is fourfold degenerate since, in addition to the polarization degeneracy, one also has the mode degeneracy of the $P_{x}$ and $P_{y}$ orbitals. It can be seen in Figs. 1(e) and 2(j) that, for the $P$ flat band, the emission predominantly has two lobes aligned along the $x$ direction on $A$ sites, corresponding to $P_{x}$ orbitals, while on the $C$ sites, the $P_{y}$ orbitals dominate. We estimate the ratio of the orbital populations to be about 6:1, corresponding to $\left|\psi_{P_{x}}\right|^{2} /\left|\psi_{P_{v}}\right|^{2}$ on $A$ sites and $\left|\psi_{P_{y}}\right|^{2} /\left|\psi_{P_{x}}\right|^{2}$ on $C$ sites [49]. As before, one needs to explain the suppression of emission from $B$ sites, since only orbitals with the same symmetry and polarization may destructively interfere. Similar to the previously described polarizationdependent tunneling probability, one can observe that particles in $P_{x}$ orbitals tunnel more easily along the $x$ direction than along the $y$ direction, and therefore, more particles in the $P_{x}$ orbital of $A$ sites are needed to satisfy Eq. (1), and the opposite holds for $P_{y}$ orbitals.

To confirm this, we also developed a TB model for the four degenerate $P$ orbitals with tunneling amplitudes that depend on the polarization and on the alignment of the mode with respect to the hopping direction [49]. As shown in Fig. 4(d), now, we have four tunneling parameters: $\tau_{\|}^{a}$, $\tau_{\perp}^{a}, \tau_{\|}^{t}$, and $\tau_{\perp}^{t}$, where $(a)$ and $(t)$ indicate whether the hopping is for $P$ orbitals aligned or transverse to the propagation direction, and $(\|)$ and $(\perp)$ indicate, as before, whether the polarization is parallel or perpendicular to the hopping direction. Similar to the $S$ band, here, also, all four modes of each pillar will be populated by the quasiresonant pump, but on $A$ sites, the population of $P_{x} H$-polarized particles will be the highest, since their probability to tunnel to $B$ sites $\left(\tau_{\perp}^{t}\right)$ is the lowest. Conversely, on $C$ sites, the population of $P_{y} V$-polarized particles will be the highest. This is exactly what is observed in Fig. 4(b). By fitting the TB band structure to the experimentally observed $P$ band and degree of linear polarization, the following hopping parameters can be obtained: $\tau_{\|}^{a}=0.375, \tau_{\perp}^{a}=0.125$, $\tau_{\|}^{t}=0.100$, and $\tau_{\perp}^{t}=0.033 \mathrm{meV}$. Note that, for the $P$ band, the difference between the hopping with $\perp$ and \| polarization is bigger than in the $S$ band case. This can be ascribed to the fact that the $P$ flat band consists of harmonics with higher $k$ values, where polarization-dependent tunneling is expected to be enhanced [45]. With the values for the hopping parameters above, we obtain a degree of polarization of the order of 0.42 and a ratio between the populations of $P_{x}$ and $P_{y}$ orbitals on $A$ sites of 4.1 (the inverse applies to $C$ sites), in good agreement with the experimental values of 0.5 and 6 [49]. It should be noted that the tunneling arguments presented here apply equally in the singleparticle regime, and as such, polarization patterns are observed in the $S$ and $P$ flat-band emission below threshold. The polarization degree of the $P$ flat band was considerably lower $(\sim 0.2)$ than in the condensate regime, however, probably due to contribution of the emission of the dispersive band at the same energy [cf. Fig. 2(g)].

In summary, we have studied the properties of a twodimensional Lieb lattice for exciton polaritons, demonstrating bosonic condensation into two separate flat bands 
formed from $S$ and $P$ orbitals, in addition to the negative effective mass states at the maxima of the $S$ antibonding band. We have also revealed distinctive emission patterns formed by the symmetric $S$ and asymmetric $P$ orbitals, which show pseudospin texture arising from spin-orbit coupling given by polarization-dependent tunneling between pillars. Our Letter shows the potential for engineering versatile lattice Hamiltonians for polaritons, highlighting the ease with which spin-orbit coupling terms and population of higher orbitals can be implemented, which presents a significant advantage of this system. Furthermore, the observation of flat-band condensate fragmentation demonstrates the effect of many-body interactions in the presence of quenched kinetic energy. An intriguing future prospect is studying quantum fluctuations as in recent polariton works [32-34], in lattice environments where novel driven-dissipative phase transitions are expected [58].

Currently, the strength of polariton-polariton interactions in a single lattice site (few $\mu \mathrm{eV}$ ) [29] is comparable to or less than the polariton decay rate. However, the ratio of these two quantities may be further enhanced via polariton Feshbach resonances [59] or recently developed high- $Q$ open-access microcavities with strong lateral confinement [44]. This would open the way to strongly correlated regimes described by driven-dissipative Bose-Hubbard models in polaritonic lattices [60]. Such regimes are not accessible in weakly coupled photonic systems.

Data supporting this study are openly available from the University of Sheffield repository [61].

The work was supported by EPSRC Grant No. EP/ N031776/1, ERC Advanced Grant EXCIPOL No. 320570 and Megagrant No. 14.Y26.31.0015. D. R. G. and I. V. I. acknowledge support from Project No. 3.8884.2017/8.9 of the Ministry of Education and Science of the Russian Federation. I. A. S. acknowledges support from Project No. 3.2614.2017 of the Ministry of Education and Science of the Russian Federation and Rannis Project No. 163082-051.

*cewhittaker1@sheffield.ac.uk

d.krizhanovskii@sheffield.ac.uk

[1] S. Zhang, H.-h. Hung, and C. Wu, Phys. Rev. A 82, 053618 (2010).

[2] C. Wu, D. Bergman, L. Balents, and S. Das Sarma, Phys. Rev. Lett. 99, 070401 (2007).

[3] Y.-F. Wang, Z.-C. Gu, C.-D. Gong, and D. N. Sheng, Phys. Rev. Lett. 107, 146803 (2011).

[4] E. H. Lieb, Phys. Rev. Lett. 62, 1201 (1989).

[5] B. Keimer, S. A. Kivelson, M. R. Norman, S. Uchida, and J. Zaanen, Nature (London) 518, 179 (2015).

[6] C. Weeks and M. Franz, Phys. Rev. B 82, 085310 (2010).

[7] N. Goldman, D. F. Urban, and D. Bercioux, Phys. Rev. A 83, 063601 (2011).

[8] W. Beugeling, J. C. Everts, and C. Morais Smith, Phys. Rev. B 86, 195129 (2012).
[9] E. J. Bergholtz and Z. Liu, Int. J. Mod. Phys. B 27, 1330017 (2013).

[10] W.-F. Tsai, C. Fang, H. Yao, and J. Hu, New J. Phys. 17, 055016 (2015).

[11] I. N. Karnaukhov and I. O. Slieptsov, arXiv:1510.07239.

[12] G. Palumbo and K. Meichanetzidis, Phys. Rev. B 92, 235106 (2015).

[13] A. Dauphin, M. Müller, and M. A. Martin-Delgado, Phys. Rev. A 93, 043611 (2016).

[14] R. Chen and B. Zhou, Chin. Phys. B 25, 067204 (2016).

[15] R. Wang, Q. Qiao, B. Wang, X.-H. Ding, and Y.-F. Zhang, Eur. Phys. J. B 89, 192 (2016).

[16] M. Di Liberto, A. Hemmerich, and C. Morais Smith, Phys. Rev. Lett. 117, 163001 (2016).

[17] D. Guzmán-Silva, C. Mejía-Cortés, M. A. Bandres, M. C. Rechtsman, S. Weimann, S. Nolte, M. Segev, A. Szameit, and R. A. Vicencio, New J. Phys. 16, 063061 (2014).

[18] S. Mukherjee, A. Spracklen, D. Choudhury, N. Goldman, P. Öhberg, E. Andersson, and R. R. Thomson, Phys. Rev. Lett. 114, 245504 (2015).

[19] F. Diebel, D. Leykam, S. Kroesen, C. Denz, and A. S. Desyatnikov, Phys. Rev. Lett. 116, 183902 (2016).

[20] C. Poli, H. Schomerus, M. Bellec, U. Kuhl, and F. Mortessagne, 2D Mater. 4, 025008 (2017).

[21] S. Taie, H. Ozawa, T. Ichinose, T. Nishio, S. Nakajima, and Y. Takahashi, Sci. Adv. 1, e1500854 (2015).

[22] D. López-González and M. I. Molina, Phys. Rev. A 93, 043847 (2016).

[23] G. Gligorić, A. Maluckov, L. Hadžievski, S. Flach, and B. A. Malomed, Phys. Rev. B 94, 144302 (2016).

[24] A. Amo and J. Bloch, C.R. Phys. 17, 934 (2016).

[25] J. Kasprzak, M. Richard, S. Kundermann, A. Baas, P. Jeambrun, J. M. J. Keeling, F. M. Marchetti, M. H. Szymanska, R. Andre, J. L. Staehli et al., Nature (London) 443, 409 (2006).

[26] R. Balili, V. Hartwell, D. Snoke, L. Pfeiffer, and K. West, Science 316, 1007 (2007).

[27] A. P. D. Love, D. N. Krizhanovskii, D. M. Whittaker, R. Bouchekioua, D. Sanvitto, S. A. Rizeiqi, R. Bradley, M. S. Skolnick, P. R. Eastham, R. André et al., Phys. Rev. Lett. 101, 067404 (2008).

[28] D. N. Krizhanovskii, K. G. Lagoudakis, M. Wouters, B. Pietka, R. A. Bradley, K. Guda, D. M. Whittaker, M. S. Skolnick, B. Deveaud-Plédran, M. Richard et al., Phys. Rev. B 80, 045317 (2009).

[29] P. M. Walker, L. Tinkler, D. V. Skryabin, A. Yulin, B. Royall, I. Farrer, D. A. Ritchie, M. S. Skolnick, and D. N. Krizhanovskii, Nat. Commun. 6, 8317 (2015).

[30] M. Sich, D. V. Skryabin, and D. N. Krizhanovskii, C.R. Phys. 17, 908 (2016).

[31] Universal Themes of Bose-Einstein Condensation, edited by N. P. Proukakis, D. W. Snoke, and P. B. Littlewood (Cambridge University Press, Cambridge, England, 2017).

[32] S. R. K. Rodriguez, W. Casteels, F. Storme, N. Carlon Zambon, I. Sagnes, L. Le Gratiet, E. Galopin, A. Lemâttre, A. Amo, C. Ciuti et al., Phys. Rev. Lett. 118, 247402 (2017).

[33] C. E. Whittaker, B. Dzurnak, O. A. Egorov, G. Buonaiuto, P. M. Walker, E. Cancellieri, D. M. Whittaker, E. Clarke, S. S. Gavrilov, M. S. Skolnick et al., Phys. Rev. X 7, 031033 (2017). 
[34] T. Fink, A. Schade, S. Höfling, C. Schneider, and A. Ímamoğlu, Nat. Phys., DOI: 10.1038/s41567-017-0020-9 (2017).

[35] N. Y. Kim, K. Kusudo, C. Wu, N. Masumoto, A. Loffler, S. Hofling, N. Kumada, L. Worschech, A. Forchel, and Y. Yamamoto, Nat. Phys. 7, 681 (2011).

[36] T. Jacqmin, I. Carusotto, I. Sagnes, M. Abbarchi, D. D. Solnyshkov, G. Malpuech, E. Galopin, A. Lemaître, J. Bloch, and A. Amo, Phys. Rev. Lett. 112, 116402 (2014).

[37] K. Winkler, J. Fischer, A. Schade, M. Amthor, R. Dall, J. Geßler, M. Emmerling, E. A. Ostrovskaya, M. Kamp, C. Schneider et al., New J. Phys. 17, 023001 (2015).

[38] E. A. Cerda-Méndez, D. N. Krizhanovskii, M. Wouters, R. Bradley, K. Biermann, K. Guda, R. Hey, P. V. Santos, D. Sarkar, and M. S. Skolnick, Phys. Rev. Lett. 105, 116402 (2010).

[39] D. N. Krizhanovskii, E. A. Cerda-Méndez, S. Gavrilov, D. Sarkar, K. Guda, R. Bradley, P. V. Santos, R. Hey, K. Biermann, M. Sich et al., Phys. Rev. B 87, 155423 (2013).

[40] E. A. Cerda-Méndez, D. N. Krizhanovskii, K. Biermann, R. Hey, M. S. Skolnick, and P. V. Santos, Phys. Rev. B 86, 100301 (2012).

[41] G. Tosi, G. Christmann, N. G. Berloff, P. Tsotsis, T. Gao, Z. Hatzopoulos, P. G. Savvidis, and J. J. Baumberg, Nat. Phys. 8, 190 (2012).

[42] M. Milićević, T. Ozawa, G. Montambaux, I. Carusotto, E. Galopin, A. Lemaître, L. Le Gratiet, I. Sagnes, J. Bloch, and A. Amo, Phys. Rev. Lett. 118, 107403 (2017).

[43] V. G. Sala, D. D. Solnyshkov, I. Carusotto, T. Jacqmin, A. Lemaître, H. Terças, A. Nalitov, M. Abbarchi, E. Galopin, I. Sagnes et al., Phys. Rev. X 5, 011034 (2015).

[44] S. Dufferwiel, F. Li, E. Cancellieri, L. Giriunas, A. A. P. Trichet, D. M. Whittaker, P. M. Walker, F. Fras, E. Clarke, J. M. Smith et al., Phys. Rev. Lett. 115, 246401 (2015).

[45] D. Solnyshkov and G. Malpuech, C.R. Phys. 17, 920 (2016).
[46] I. A. Shelykh, A. V. Kavokin, Y. G. Rubo, T. C. H. Liew, and G. Malpuech, Semicond. Sci. Technol. 25, 013001 (2010).

[47] F. Baboux, L. Ge, T. Jacqmin, M. Biondi, E. Galopin, A. Lemaître, L. Le Gratiet, I. Sagnes, S. Schmidt, H. E. Türeci et al., Phys. Rev. Lett. 116, 066402 (2016).

[48] B. Sutherland, Phys. Rev. B 34, 5208 (1986).

[49] See Supplemental Material at http://link.aps.org/ supplemental/10.1103/PhysRevLett.120.097401 for further details about the sample, experiment, and theoretical models, which includes Ref. [50].

[50] D. Bajoni, P. Senellart, A. Lemaître, and J. Bloch, Phys. Rev. B 76, 201305 (2007).

[51] E. L. Shirley, L. J. Terminello, A. Santoni, and F. J. Himpsel, Phys. Rev. B 51, 13614 (1995).

[52] M. Niţă, B. Ostahie, and A. Aldea, Phys. Rev. B 87, 125428 (2013).

[53] D. N. Krizhanovskii, D. Sanvitto, A. P. D. Love, M. S. Skolnick, D. M. Whittaker, and J. S. Roberts, Phys. Rev. Lett. 97, 097402 (2006).

[54] D. Tanese, H. Flayac, D. Solnyshkov, A. Amo, A. Lemaître, E. Galopin, R. Braive, P. Senellart, I. Sagnes, G. Malpuech et al., Nat. Commun. 4, 1749 (2013).

[55] E. A. Cerda-Méndez, D. Sarkar, D. N. Krizhanovskii, S. S. Gavrilov, K. Biermann, M. S. Skolnick, and P. V. Santos, Phys. Rev. Lett. 111, 146401 (2013).

[56] S. D. Huber and E. Altman, Phys. Rev. B 82, 184502 (2010).

[57] A. V. Nalitov, G. Malpuech, H. Terças, and D. D. Solnyshkov, Phys. Rev. Lett. 114, 026803 (2015).

[58] A. Biella, F. Storme, J. Lebreuilly, D. Rossini, R. Fazio, I. Carusotto, and C. Ciuti, Phys. Rev. A 96, 023839 (2017).

[59] N. Takemura, S. Trebaol, M. Wouters, M. T. Portella-Oberli, and B. Deveaud, Nat. Phys. 10, 500 EP (2014).

[60] R. Rota, W. Casteels, and C. Ciuti, Eur. Phys. J. Spec. Top. 226, 2805 (2017).

[61] https://figshare.com/articles/Data_for_manuscript_Exciton_ Polaritons_in_a_Two-Dimensional_Lieb_Lattice_with_SpinOrbit_Coupling/5868084. 